and globally accessible KT approach. The MOOC also successfully combined primary prevention with more traditional secondary and tertiary concussion prevention foci, thereby enhancing programming that aims to improve concussion literacy.

\section{IS IT POSSIBLE TO STIMULATE INJURY-PREVENTIVE BEHAVIOUR IN ADULT NOVICE RUNNERS WITH AN ONLINE INTERVENTION? RESULTS OF A RANDOMIZED CONTROLLED TRIAL}

Ellen Kemler, Maaike Cornelissen, Vincent Gouttebarge. Dutch Consumer Safety Institute, Amsterdam, Netherlands

\subsection{6/bjsports-2021-IOC.22}

Background In addition to beneficial health effects, running is associated with a risk of sustaining injuries. The online intervention Runfitcheck was developed to stimulate injury-preventive behaviour among adult novice runners.

Objective To evaluate the effectiveness of Runfitcheck on injury-preventive behaviour among adult novice runners.

Design Randomized controlled trial.

Setting Participants were recruited via Dutch social media networks. Participants were asked to fill in four online questionnaires.

Participants The group of participants consisted of adult novice runners.

Interventions (or Assessment of Risk Factors) The intervention group had access to the Runfitcheck intervention, while the control group performed their running activities as usual.

Main Outcome Measurements Injury-preventive behaviour: (i) using a (personalised) training schedule; (ii) performing strength and technique exercises; and (iii) performing a warmup routine prior to running. Relative Risks ((RR) and 95\% Confidence Interval $(95 \% \mathrm{CI})$ ) were used to analyse behavioural change among runners who were not performing the favourable behaviour at enrolment.

Results A total of 1,411 novice runners $(72.6 \%$ female, mean age 38.1 years) were included. Runners in the intervention group searched more often for information about a warm-up routine $(53.6 \%$ versus $33.6 \%$; $n=194$; RR 1.444 (95\%CI 1.098-1.901)), performed a regular warm-up routine more often $(47.1 \%$ versus $28.4 \% ; n=196 ; R R \quad 1.461 \quad$ (95\%CI 1.084-1.968), and added strength exercises to their warm-up routine more often than runners in the control group (32.6\% versus $17.4 \%$; $n=192$; RR 1.504 (95\%CI 1.0392.179)). Runners in the intervention group performed strength exercises less often at other points of time during the week (other than during their warm-up routine) than runners in the control group $(36.6 \%$ versus $50.0 \% ; n=426$; RR 0.790 (95\%CI 0.669-0.932)). No significant results were found for using a training schedule and running technique exercises.

Conclusions The online intervention Runfitcheck was effective in stimulating aspects of injury-preventive behaviour in adult novice runners related to a warm-up routine, but did not succeed in stimulating other aspects of injury-preventive behaviour.

\section{DIFFERENCES IN INJURY CHARACTERISTICS BETWEEN ATHLETICS DISCIPLINES DURING INTERNATIONAL ATHLETICS CHAMPIONSHIPS}

1,2,3,4,5 Pascal Edouard, 6 Laurent Navarro, ${ }^{5}$ Pedro Branco, 4,7Vincent Gremeaux ${ }^{8}$ Toomas Timpka, ${ }^{9,10}$ Astrid Junge. ${ }^{1}$ Inter-University Laboratory of Human Movement Science (LIBM EA 7424), University of Lyon, University Jean Monnet, Saint-Etienne, France; ${ }^{2}$ Department of Clinical and Exercise Physiology, Sports Medicine Unit, University Hospital of Saint-Etienne, Faculty of Medicine, Saint-Etienne, France; ${ }^{3}$ Medical Commission, French Athletics Federation (FFA), Paris, France; ${ }^{4}$ Swiss Olympic Medical center, Centre de médecine du sport, Division de médecine physique et réadaptation, Centre Hospitalier Universitaire Vaudois, Lausanne, Switzerland; ${ }^{5}$ European Athletics Medical and Anti Doping Commission, European Athletics Association (EAA), Lausanne, Switzerland; ${ }^{6}$ Mines SaintEtienne, Univ Lyon, Univ Jean Monnet, INSERM, U 1059 Sainbiose, Centre CIS, SaintEtienne, France; ${ }^{7}$ Institute of Sport Sciences, University of Lausanne, Lausanne, Switzerland; ${ }^{8}$ Athletics Research Center, Linköping University, Linkoping, Sweden; ${ }^{9}$ Medical School Hamburg, Hamburg, Germany; ${ }^{10}$ Swiss Concussion Center and Schulthess Clinic Zürich, Zurich, Switzerland

\subsection{6/bjsports-2021-IOC.23}

Background Competing in international athletics championships bears the risk of injury. Athletics is composed of different disciplines with different physical, mechanical, technical and psychological demands, leading to different injury rates according to disciplines. However, the specific injury characteristics according to disciplines have not been yet described for high-level athletes during international championships.

Objective To analyse differences in injury characteristics between athletics disciplines during international athletics championships.

Design Prospective study.

Setting 14 international championships from 2007 to 2018.

Participants 8925 male and 7614 female registered international-level athletes.

Main outcome measurements The national medical teams and the local organizing committee physicians reported all newly incurred injuries daily on a standardised injury report form, including the characteristics of each injury: location, type, cause and severity. We analysed differences in injury characteristics distribution between the nine disciplines separately for male and female athletes using $\mathrm{Chi}^{2}$-tests, or Fisher' exact test where appropriate.

Results A total of 928 injuries were reported in male and 597 in female athletes. Injury characteristics significantly varied between disciplines for location, type, cause and severity, in both male and female athletes. Thigh muscle injuries were the main injury diagnoses in sprints, hurdles, jumps, combined events and race walking, lower leg muscle injuries in marathon, lower leg skin injury in middle and long distances, and trunk muscle and lower leg muscle injuries in throws.

Conclusions Short-distance running disciplines exposed to thigh muscle injuries, while long-distance running disciplines exposed to lower leg muscle injuries, and disciplines involving plyometric (i.e. jumps or combined events) are associated with musculoskeletal injuries of different locations and types. Strategies for medical service provision, including local organisation, medical teams, supplies, and facilities, in the preparation of and at athletics championships should be discipline-specific and prepared for targeting the main injuries in each discipline as they appear in the program. 